\title{
How College Students Assess the Threat of Infectious Diseases: Implications for University Leaders and Health Communicators
}

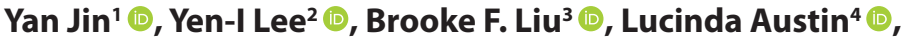 \\ and Seoyeon $\mathrm{Kim}^{5}$ (D)
}

1. Grady College of Journalism and Mass Communication, University of Georgia, Athens, GA, USA

2. Murrow College of Communication, Department of Strategic Communication, Washington State University, Pullman, WA, USA

3. Department of Communication, University of Maryland, College Park, MD, USA

4. Hussman School of Journalism and Media, University of North Carolina at Chapel Hill, Chapel Hill, NC, USA

5. College of Communication and Information Sciences, University of Alabama, Tuscaloosa, AL, USA

\section{ABSTRACT}

Higher education institutions and their students face a wide range of infectious disease threats (IDTs). However, there is a lack of theory-driven research on how to provide communication for multiple IDTs to motivate protective action taking. To close this gap, this study focuses on college students and two IDT types: respiratory and sexually transmitted infections. We tested an IDT appraisal model with data from an online survey conducted at two U.S. universities with 842 students. Findings indicate that IDT type led to different patterns of threat appraisal and protective action taking intentions. More specifically, participants perceived sexually transmitted threats as significantly more predictable and more controllable than respiratory threats.

Participants also had higher intention to take protective action in response to respiratory threats than sexually-transmitted threats. We also found that externalattribution-dependent (EAD) emotions (i.e., anger, sadness, surprise, and confusion)

CONTACT Yan Jin (D) - E-mail: yanjin@uga.edu • Grady College of Journalism and Mass Communication • University of Georgia • 120 Hooper Street • Athens, GA 30602, USA 
and an internal-attribution-dependent (IAD) emotion (i.e., hope) were sequential mediators in the relationship between IDT appraisal and protective action taking intentions for both infectious disease types. Implications for IDT communication research and practice are discussed.

KEYWORDS: infectious disease, threat appraisal, crisis emotions, higher education

Higher education institutions and their students have increasingly faced infectious disease threats (IDTs). These threats have included the $2009 \mathrm{H}_{1} \mathrm{~N}_{1}$ outbreak, Middle East respiratory syndrome (MERS-CoV) outbreaks, annual exposure to seasonal influenza (Y-I. Lee et al., 2018), and frequent exposure to sexually transmitted infections including human papillomavirus or HPV (Alsulaiman \& Rentner, 2018; Yang \& Pittman, 2017; L. Zhang et al., 2015). Notably, thousands of U.S. higher education institutions are currently navigating how to respond to the coronavirus pandemic, and early evidence suggests that these institutions are struggling in their risk communication about COVID-19 (Burke, 2020). Compared to research on active shooter incidents and natural disasters, students' physical and emotional health during IDTs has not been extensively explored (Moerschell \& Novak, 2020). This study builds on recent trends to develop and test a new theoretical model tailored for infectious disease crisis communication (Jin et al., 2020; Y-I. Lee \& Jin, 2019; B. F. Liu et al., 2020).

In the field of health communication, there has been a substantial body of literature on sexually transmitted infections (STIs), often testing messages to promote healthy behaviors among college students (e.g., Boudewyns \& Paquin, 2011; Lin \& Lagoe, 2013; Yang, 2015). Indeed, every year almost half of the 20 million newly diagnosed STIs in the U.S. are among young adults aged 15 to 24 (CDC, 2016). Likewise, every year college campuses face the threat of seasonal flu outbreaks (Y-I. Lee et al., 2018). Accordingly, college student samples are ideal for research on IDTs. However, despite the prevalence of IDTs on campus, there is little research examining how college students can positively navigate these threats. Instead, the preponderance of research examines a single IDT (e.g., Best et al., 2018; Rubin et al., 2009; Taha et al., 2013; Yang \& Pittman, 2017). 
This study builds on prior research developing a new theoretical model tailored for infectious disease crisis communication (Jin et al., 2020; Y-I. Lee \& Jin, 2019; B. F. Liu et al., 2020), factoring in the impact of negative and positive crisis emotions most relevant to IDTs (Jin et al., 2020; Jin et al., 2014; B. F. Liu et al, 2016; van der Meer \& Jin, 2020). Our approach contributes to health risk and crisis communication research on at least two fronts. First, we examine how college students respond to different IDTs, controlling for individual differences. Second, we evaluate affect, integrating discrete emotions, and affective dimensionality into the health risk communication literature.

We focus on college students and two types of IDTs: (1) respiratory infectious diseases, given the enormous impact of COVID19 on campuses around the world, and (2) the long-standing threat of STIs among this population. We tested the proposed model with data from an online survey conducted at two U.S. universities with 842 students conducted in 2017. Findings reveal that IDT type (i.e., respiratory diseases versus STIs) led to different patterns of IDT appraisal and protective action taking intentions. Moreover, among identified IDT attribution-dependent emotions, external-attribution-dependent (EAD) emotions (i.e., anger, sadness, surprise, and confusion) and internal-attribution-dependent (IAD) emotion (i.e., hope) were found to be sequential mediators in the relationship between IDT appraisal and protective action taking intentions for both IDTs.

\section{Literature Review}

\section{Infectious Disease Threat (IDT) Appraisal}

Grounded in Jin's (2010) cognitive appraisal model of crises and risks and its three primary appraisal dimensions (i.e., perceived predictability, controllability, and responsibility), scholars have recently developed a new IDT appraisal model (Jin et al., 2020), containing three key dimensions of how publics appraise IDTs: perceived predictability, controllability, and responsibility. The initial empirical examination was based on a representative sample of U.S. adults. This prior study revealed that the IDT appraisal model helped explain and predict individuals' cognitive, emotional, and 
behavioral responses to sexually transmitted infections and threats from waterborne, foodborne, and vector borne diseases. The results supported the IDT appraisal model's overarching proposition: Individuals' assessments of predictability, controllability, and responsibility drive their affective responses, information seeking, and conative reactions to IDTs. This study builds on the initial IDT appraisal model research in multiple ways. First, this study investigates the role of discrete emotions beyond affective valence in the process of how individuals appraise IDTs and the impact of this appraisal process on behavioral outcomes.

Second, this study uncovers whether the IDT appraisal model can be applied to specific at-risk populations (e.g., college students) in the context of two threats: STIs and respiratory diseases. Therefore, this study applies the new IDT appraisal model (Jin et al., 2020) to examine how college students in the U.S. appraise some of the most challenging and common IDTs (i.e., respiratory and STIs). Findings reveal how students' appraisals of IDT predictability, controllability, and responsibility predict students' affective responses and intentions to take protective actions.

It is imperative for college students to take recommended actions to protect themselves against STIs (e.g., wear a condom, get vaccinated) and respiratory diseases (e.g., wash hands, maintain social distance, cover coughs/sneezes, get vaccinated). In this section, we review the growing body of literature linking IDT predictability, controllability, and responsibility to protective action taking in response to STIs and respiratory diseases.

\section{Perceived IDT Predictability}

Individuals' risk perceptions vary by subjective judgment of different characteristics (e.g., whether a risk is dreadful or familiar) associated with risk issues (Ropeik, 2002; Slovic, 1987). In an IDT situation, perceived predictability is defined as the extent to which individuals perceive that they can predict what will happen (Jin, 2010; Jin et al., 2020).

A recent study found that individuals' assessments of the predictability of an IDT situation drove their intentions to take protective actions (Jin et al., 2020). No prior research has examined the relationship between perceived IDT predictability and college 
students' intentions to take protective actions against respiratory diseases and STIs. However, research has consistently found that college students often display an optimistic bias in that they do not actively engage in preventative behaviors to mitigate their risk of contracting infectious diseases (Afifi \& Weiner, 2006; Best et al., 2018). Given the dearth of prior research comparing college students' responses to respiratory and sexually transmitted IDTs, this study asks:

RQ1.1: Do college students perceive IDT predictability differently across respiratory and sexually transmitted IDTs, and if so, how?

\section{Perceived IDT Controllability}

Jin (2010) conceptualized control as individuals' beliefs that human agency is present or available for a crisis or risk situation. In an IDT situation, perceived controllability is connected to a sense that treatments or prevention of an infectious disease are possible or available (Jin et al., 2020). For example, novel and severe infectious diseases may be seen by the public as low in controllability (Ropeik, 2002; Slovic, 1987) due to the lack of an available vaccine, evolving scientific knowledge, or disease containment uncertainty.

Risk perception has been linked to perceptions of the controllability of health and safety risks among college students (Inungu et al., 2009; Weinstein, 1984). Alarmingly, college students are unrealistically optimistic about their risk of contracting diseases that are perceived as preventable through personal action (i.e., controllable) (Weinstein, 1984). Research is mixed when it comes to whether risk perception of contracting a respiratory infectious disease predicts vaccination intentions among U.S. college students. Some research finds that students do not believe that an infectious disease threat is severe enough to merit vaccination (Cornally et al., 2013; Roberto et al., 2019). Other research finds that messages of self-efficacy that highlight benefits of vaccines can motivate college students to vaccinate (Agarwal, 2014; Yang, 2015).

In the context of STIs, one study found that college students were more likely to report taking protective actions (i.e., condom use) if they viewed STIs as having highly negative impacts on their health (Rintamaki \& Yang, 2013). However, they only used 
condoms if they believed that condoms were effective at reducing their risk of contracting an STI (Rintamaki \& Yang, 2013). Similarly, in the context of HPV, one study found that the shame of contracting HPV and perceptions of high controllability predicted intentions to vaccinate among college students if the vaccine was available immediately at no cost (Yang \& Pittman, 2017). Given the mixed research record, this study further investigates how perceptions of IDT controllability influence college students' decisions to take protective actions against respiratory diseases and STIs. Therefore, we ask:

RQ1.2: Do college students perceive IDT controllability differently across respiratory and sexually transmitted IDTs, and if so, how?

\section{Perceived IDT Responsibility}

As acknowledged in the development of the IDT appraisal model (Jin et al., 2020), there is a gap between how crisis and health scholars define and measure responsibility in different contexts (e.g., organizational crisis versus public health crisis). Crisis communication scholars have defined crisis responsibility as "the degree to which stakeholders blame the organization for the crisis event" (Coombs, 1998, p. 180), which can be observed by the degree of blame placed on an organization and can lead to negative organizational outcomes (e.g., Coombs \& Holladay, 2005; W. Liu et al., 2018). Given that an IDT situation is in the domain of public health, it is critical to examine individuals' perceived IDT responsibility perceptions through the lens of health communication.

According to the IDT appraisal model (Jin et al., 2020), IDT responsibility is based on the integration of public health and crisis communication literature and includes two facets of responsibility (Jin et al., 2020; Y. Zhang et al., 2015): the responsibility for the cause(s) of threats and the potential solutions. Regarding the organizational facet of IDT responsibility, although people are unlikely to hold a health authority responsible for actively spreading a virus (IDT cause), they are likely to hold authorities responsible for perceived lack of effective prevention and control of the disease (IDT solution) (Jin et al., 2020). We posit that this definition will apply 
to the population of college students and the two specific IDT types we examine in this study.

In terms of the IDT responsibility facet, research connects personal responsibility with protective actions in the context of STIs (Best et al., 2018; Boudewyns \& Paquin, 2011; Cheah, 2006; Vorpahl \& Yang, 2018) and respiratory infectious diseases (Panda et al., 2015). One study found that patients aged 17 to 27 displayed difficulty connecting HPV and their cancer diagnosis. Consequently, they rarely made connections between preventative behaviors they could take in the future and actions that they could recommend to their friends and family members (Best et al., 2018). Other research has echoed these findings, noting a "tremendous lack of awareness by college students about the sexual realities that they face" (Afifi \& Weiner, 2006, p. 49).

To improve awareness, one study found that college students believe that campus health centers have a responsibility for educating them about STIs (Cheah, 2006). Campaigns might be most effective when they persuade college students that getting tested for STIs would show respect for their sexual partners and prevent the spread of an STI to someone else (Boudewyns \& Paquin, 2011). Conversely, U.S. college students may be receptive to messages that attribute the cause of HPV infection as external (i.e., others can pass HPV on to you) rather than internal (i.e., you can pass HPV on to others), and messages with external attributions led to a higher vaccine intentions (Vorpahl \& Yang, 2018).

In regard to responsibility perceptions associated with respiratory infectious diseases, the global community has witnessed people blaming individuals living in the region where a new infectious disease was first reported (Schram, 2003; Washer, 2004) and being suspicious about transparency and credibility of the guidelines provided by their governments and other health institutions (Lau et al., 2003; Pickles \& Goodwin, 2006). Given the highly uncertain and more easily spreading nature of respiratory infectious diseases, the public expects governments and health authorities to take responsibility for providing prompt and appropriate guidelines (Smith, 2006). Indeed, a lesson learned from past management of respiratory IDTs (e.g., SARS, MERS-CoV) was that responsibility, 
authority, and accountability should be clearly communicated to effectively respond to IDTs (Smith, 2006). Also, public health professionals have emphasized that it is critical to create an atmosphere of mutual trust and solidarity when communicating respiratory IDT information for the public to follow health authorities' guidelines with confidence (Kotalik, 2005; S. I. Lee, 2015).

Based on the literature reviewed above, it is important to understand individuals' perceived responsibility for the cause and solution of an IDT in order to effectively intervene. Therefore, this study examines how attributed blame to at-risk individuals, health professionals, and health authorities might impact protective action decisions. Therefore, we ask:

RQ1.3: Do college students perceive IDT responsibility differently across respiratory and sexually transmitted IDTs, and if so, how?

\section{IDT Appraisal and Protective Action Taking}

The recent development of the IDT appraisal model based on a general U.S. adult sample suggests that individuals' perceived IDT predictability, controllability, and responsibility impact how they respond to an IDT situation across different IDT types (Jin et al., 2020). For example, Jin and colleagues (2020) found that more predictable but less controllable IDTs led to increased information seeking on specific media channels (e.g., government social media) as well as protective behaviors. Furthermore, IDTs that were less predictable and less controllable were associated with more information seeking and protective behavioral intentions.

When individuals have a good understanding about an IDT (high predictability) and feel that they have volitional control over the situation (high controllability), the overall perceived threat is likely to be low (de Zwart et al., 2009; Weinstein, 1984). Given the low perceived threat, individuals may become optimistic about their susceptibility to a given infectious disease and are less likely to engage in protective behaviors (e.g., de Zwart et al., 2009; Inungu et al., 2009). Studies have found that protective action taking is more likely to occur when the perceived threat is high and an individual has self-efficacy or the belief that one has volitional control over a situation (Floyd et al., 2000; Kim \& Hawkins, 2020; Witte, 1992). 
STIs are probably perceived as being more predictable and controllable, compared to respiratory infectious diseases, which are often newly emerging diseases with causes and treatment unknown. When exposed to respiratory IDTs, especially during the initial phase, individuals' anxiety for unknown risks is likely to increase and, consequently, they may engage in protective action taking (Lau et al., 2003; Leung et al., 2005; Rubin et al., 2009). However, little is known about how responsibility perceptions are related to protective actions taken in response to different IDTs. The relationship between the way an IDT is perceived in multiple ways and protective action taking needs to be further examined across different IDTs.

Applying the IDT appraisal model to understand U.S. college students' responses, we further focus on the protective actions that college students intend to take against respiratory and sexually transmitted IDTs, as protective action taking is among the most important responses to predict in order to save lives (Jin et al., 2020). We ask:

RQ2: Do college students' intention to take protective actions differ across respiratory and sexually transmitted IDTs, and if so, how?

\section{The Role of Emotions in Responding to IDT}

Emotions play a critical role in crisis and risk communication. Studies have found that emotions impact how individuals process crisis information (Jin, 2010; Jin et al., 2010) and individuals' protective action decision-making (B. F. Liu et al., 2020). Initial testing of the IDT appraisal model has found that individuals' affective responses varied by different levels of IDT appraisal dimensions (Jin et al., 2020). These affective responses further predicted behavioral outcomes (Jin et al., 2020). Affective responses may also mediate the relationship between college students' IDT appraisal and behavioral intentions in the context of respiratory and sexually transmitted IDTs.

The connection between IDT appraisal dimensions and affective responses was observed in Jin and colleagues' (2020) study. Specifically, the more predictable or controllable an IDT was perceived to be, the less likely individuals were to feel negative about 
the situation. Furthermore, total affective negativity increased toward an unpredictable IDT situation when a person or organization was viewed as being responsible for what happened. Jin and colleagues (2020) pointed out that a sense of losing control of an IDT situation could contribute to increased feelings of negative affect. To extend the affective front of the new IDT appraisal model, this study further explored how the three IDT dimensions of predictability, controllability, and responsibility might connect with discrete emotions (Jin et al., 2014).

Furthermore, a variety of studies have shown a connection between certain emotions and individuals' protective actions taken against respiratory infectious diseases. Specifically, research has connected fear to protective action taking, such as reduced contact with friends, avoidance of social gatherings, and intentions to vaccinate among adults (Cowling et al., 2010; Guo et al., 2005; Leung et al., 2005). Another study with U.S. college students found that media consumption predicted protective behaviors, such as hand-washing and vaccination intentions in response to respiratory infectious diseases, mediated by fear and knowledge (L. Zhang et al., 2015). Research has also demonstrated a positive relationship between anxiety and protective action taking (Leung et al., 2005; Rubin et al., 2009). For example, a longitudinal study among Hong Kong residents found that anxiety of contracting SARS predicted reported adoption of personal protective measures, such as wearing a face mask (Leung et al., 2005). Other research also has found a link between hope and reaching personal health choices such as the selection of sexual partners (Barnett et al., 2015; Snyder et al., 1996).

However, not all research links emotions to effective protective action taking. For example, research has found that Canadian adults who engaged in emotion-focused coping versus problem-focused coping were less likely to report intentions to obtain the $\mathrm{H}_{1} \mathrm{~N}_{1}$ vaccine (Taha et al., 2013). Likewise, in a survey of Hong Kong adults during the $\mathrm{H}_{1} \mathrm{~N}_{1}$ outbreak, higher anxiety was associated with greater social distancing, but less use of hygienic measures (Cowling et al., 2010). In terms of STIs, a meta-analysis found that anxiety was strongly and positively correlated with uncertainty of STI risk; also, uncertainty was significantly associated with 
avoidance behaviors, such as not taking appropriate protective actions (Kuang \& Wilson, 2017). Therefore, more research is warranted to uncover how emotions affect decisions to take protective actions in response to respiratory diseases and STIs.

\section{IDT Emotions by the Locus of Attribution}

The level of responsibility attributed to an event (e.g., a health crisis) is associated with the types of emotions the individual feels about the event (Coombs \& Holladay, 2005; Jin et al., 2010). Choi and Lin (2009) suggested that attribution-independent and attribution-dependent emotions may coexist during a crisis by examining emotional responses to Mattel's product recall in 2007. Jin and colleagues (2014) further identified three types of crisis emotions based on the presence and direction of attribution: (1) attribution-independent (AI) emotions (i.e., anxiety, fear, apprehension, and sympathy); (2) external-attribution-dependent (EAD) emotions (i.e., disgust, contempt, anger, and sadness); and (3) internal-attribution-dependent (IAD) emotions (i.e., guilt, embarrassment, and shame). These crisis emotions (negative and positive) co-exist and exert varied levels of influence at a given point of time as well as evolve and change over time sequentially or concurrently (Jin et al., 2014). Later crisis emotion studies suggested additional discrete emotions (e.g., surprise, confusion, hope) to affective measures, especially examining emotional responses to disasters (e.g., Jin et al., 2016) and health communication (e.g., Nabi \& Prestin, 2016; van der Meer \& Jin, 2020). In light of the new IDT appraisal model (Jin et al., 2020), we further examine IDT emotions, which are selected from Jin et al.s (2014) crisis emotion inventory. This inventory identified the positive and negative emotions that individuals are likely to feel according to whom they hold responsible for a crisis.

As Jin and colleagues (2020) uncovered, IDT appraisal dimensions and individuals' affective responses are connected. When individuals strongly believed that someone or an organization is responsible for an IDT, they were more likely to experience stronger negative emotions (Jin et al., 2020). Additionally, an IDT with higher predictability or higher controllability lowered negative 
feelings about the situation, while a sense of losing control of an IDT situation increased negative affect (Jin et al., 2020). These new insights in IDT appraisal research seem to point to the unique nature of IDT attribution, jointly affected by IDT responsibility as well as IDT predictability and controllability.

In this study, emphasizing the appraisal process and the unique nature of IDT attribution, we propose the following two attribution-dependent IDT emotions to be used in assessing college students' affective responses to respiratory and sexually transmitted IDT situations. First, EAD emotion (i.e., anger, sadness, surprise, confusion) is a predominately negative affect resulting from attributing IDT responsibility externally. For example, one is angry with a responsible party other than oneself, one feels sad because of the uncertainty of the situation, and one is surprised or confused by the situation. Second, IAD emotion (i.e., hope) is a positive affect resulting from attributing IDT responsibility internally. For example, hope has been associated with stronger self-efficacy and stronger acceptance of HPV vaccine messages among college students (Nabi \& Prestin, 2016). When it comes to infectious disease outbreak communication, a recent study found that U.S. adults are more likely to take recommended protective actions when they feel more optimistic about public health crisis situations (van der Meer \& Jin, 2020). Therefore, after initial health message exposure, if one is hopeful and optimistic that a situation can be dealt with by taking one's own responsibility, one may be more likely to take protective actions as recommended. In this way, hope as an IAD emotion can function as a mediator between health messages and intended behavioral outcomes, as in previous health studies.

\section{IDT Emotions as Sequential Mediators}

According to the Centers for Disease Control and Prevention (CDC, n.d.), infectious diseases are illnesses caused by germs that enter the human body and cause an infection. Additionally, the CDC (n.d.) has stated that some infectious diseases are contagious and spread from one individual to another; other infectious diseases are spread in the air, water, or food. People can feel threatened when they face any infectious disease threat, which can trigger negative attribution-based emotions (Jin et al., 2020). This 
study tests emotions because emotions shift in response to health messages (Nabi, 2015). Individuals experience different emotional shifts as they appraise and reappraise messages (Nabi, 2015; Nabi \& Myrick, 2019). Hope, in particular, has been identified as a positive emotion to explain how emotions flow in response to information about health risks, but negative emotional flow also occurs (Nabi, 2015). Based on attribution-based crisis emotions (Jin et al., 2014) and emotional flow (Nabi, 2015), this study examines whether and how people's negative emotions (e.g., anger, sadness, contempt, and disgust) and positive emotions (e.g., hope) flow in an IDT context. A recent study reported both positive and negative affect among individuals varied as a function of their IDT appraisal (i.e., IDT predictability and controllability), which further predicted other response outcomes such as information seeking and following recommended protective actions (Jin et al., 2020). Based on this prior research, we posit that both EAD emotions (i.e., anger, sadness, surprise, confusion) and IAD emotion (i.e., hope) function as mediators for the relationship between college students' IDT appraisals and their protective action taking intentions.

Additionally, the literature suggests a sequential relation between EAD and IAD emotions. According to Jin et al's (2014) argument, EAD emotions in general are generated due to the negative outcomes of a crisis event, which result in publics' efforts to seek the cause of these negative outcomes (Choi \& Lin, 2009; Jin et al., 2014; Weiner, 1986). IAD emotions are typically triggered according to "how individuals felt about themselves as publics associated with a given organization after learning about the crisis situation" (Jin et al., 2014, p. 512), which indicates the importance of personal identification with crisis responsibility. Given that an IDT situation is triggered by an external threat, which is likely to drive external attribution first (thus EAD emotion) and then trigger internal attribution (thus IAD emotion), we posit the following set of hypotheses, delineating an EAD-IAD sequential mediation model in the current study:

H1: EAD emotion (i.e., anger, sadness, surprise, confusion) and IAD emotion (i.e., hope), respectively, function as sequential mediators in the relationship between college students' appraisal of a respiratory IDT and protective action taking intention. 
H2: EAD emotion (i.e., anger, sadness, surprise, confusion) and IAD emotion (i.e., hope) respectively, function as sequential mediators in the relationship between college students' appraisal of a sexually transmitted IDT and protective action taking intention.

\section{Method}

An online survey was conducted to investigate how college students from two universities differently appraise the levels of predictability, controllability, and responsibility of two types of IDTs. This study also examined the hypothesized sequential-mediator roles of EAD and IAD emotions, respectively, in the relationship between college students' IDT appraisals and intentions to take protective actions.

\section{Participants and Procedures}

A total of 842 U.S. college students participated in the online study, via a participant pool system at a large Eastern public university and a large Southeastern public university in the U.S. The data collection was completed in 2017, prior to the COVID-19 pandemic. There were 299 males (35.5\%), 531 females (63.1\%), six who identified as other $(0.7 \%)$, and six preferring not to answer (0.7\%). Among participants who reported their race/ethnicity, there were 591 Caucasian (70.2\%), 83 Asian/Pacific Islander (9.9\%), 71 African American/Black (8.4\%), 59 Hispanic/Latino (7.0\%), 25 who identified as other (3.0\%), and 13 preferring not to answer (1.5\%).

Participants read a set of scenarios about two hypothetical IDT types (i.e., respiratory disease and STI). The presentation order of the scenarios was randomized as well. Participants were told about how the given disease is spread (e.g., people with the disease expel droplets of the pathogen into the air when coughing, sneezing, or talking, and others nearby may breathe in or inhale these pathogens) and that the disease could cause mild to severe illness, and at times could lead to death. A list of signs and symptoms were also provided. Although each scenario was written in a slightly different manner to prevent the participant from recognizing the experimental manipulation, disease descriptions (e.g., severity 
level, symptoms), other than the IDT type, were consistent across the two scenarios. After reading each scenario, participants completed a questionnaire that contained the measures detailed in the following section.

The study protocols were approved by the Institutional Review Boards where college student participants were recruited via the SONA system, an existing participant pool system that included all students enrolled in undergraduate classes that would grant extra credit for their research participation at the two universities. Students logged onto the SONA system to learn about potential research opportunities, where they saw our recruitment script and decided whether to participate. Students must have been 18 years of age or older to be eligible for this study. After checking a box to indicate they have read the consent form, participants then began the online survey, including scenario reading and questionnaire responding. All students who participated in this study received extra credit for their participation from their enrolled classes.

\section{Measures}

The questionnaire included items to assess participants' perceived predictability, controllability, and responsibility of each IDT, and protective action taking intentions, respectively. Participants answered the same set of questions separately for respiratory and sexually transmitted IDTs.

\section{Perceived IDT Predictability}

Perceived predictability was assessed using five items adapted from previous studies (Brummette \& Sisco, 2015; Jin, 2010) on a 7-point Likert scale, ranging from 1 "strongly disagree" to 7 "strongly agree." Participants reported to what extent different agencies would be able to predict what will happen in the given IDT situation, including individuals, federal health organizations (e.g., CDC), state health organizations (e.g., state departments of health), local health organizations (e.g., county departments of health), and medical and health professionals (e.g., physicians, nurses, and/or pharmacists). An index of perceived IDT 
predictability with averaged scores was created for respiratory IDT $(\alpha=.87, M=4.45, S D=1.36)$ and for sexually transmitted IDT $(\alpha=.86, M=4.72, S D=1.33)$.

\section{Perceived IDT Controllability}

Perceived controllability was assessed using five items adapted from previous studies (Brummette \& Sisco, 2015; Jin, 2010) on a 7-point Likert scale, ranging from 1 "strongly disagree" to 7 "strongly agree." Participants reported to what extent different entities would be able to influence what will happen in the given IDT situation, including individuals, federal health organizations (e.g., CDC), state health organizations (e.g., state departments of health), local health organizations (e.g., county departments of health), and medical and health professionals (e.g., physicians, nurses, and/or pharmacists). An index of perceived IDT controllability with averaged scores was created for respiratory $(\alpha=.87$, $M=4.62, S D=1.36)$ and for sexually transmitted IDTs $(\alpha=.83$, $M=4.82, S D=1.30)$.

\section{Perceived IDT Responsibility}

Perceived IDT responsibility (i.e., whom to blame for the IDT situation), conceptualized as combined responsibility of individuals, health professionals, and health organizations at local, state, and federal levels, was assessed using five items adapted from Coombs and Holladay's (2005) study on a 7-point Likert scale, ranging from 1 "strongly disagree" to 7 "strongly agree." The statements included "the blame for the event of [respiratory/sexually transmitted] disease threat lies with": "individuals in the circumstance," "federal health organizations (e.g., CDC)," "state health organizations (e.g., state departments of health)," "local health organizations (e.g., county departments of health)," and "medical and health professionals (e.g., physicians, nurses, and/or pharmacists)." An index of perceived IDT responsibility with averaged scores was created for respiratory $(\alpha=.92, M=3.50, S D=1.63)$ and for sexually transmitted IDTs $(\alpha=.88, M=3.85, S D=1.50)$. 


\section{Protective Action Taking Intention}

A 10-item measure of protective action taking intention adopted from Liu et al's (2016) study was presented for participants to respond using a 7-point Likert scale where " $1=$ strongly disagree" and " $7=$ strongly agree." Some of the items included: "I would follow health organizations' instructions step by step," "I would seek medical professionals' advice before deciding to follow any health organizations' instructions," and "I would listen for more information from health organization sources." An index of protective action taking intention with averaged scores was created for respiratory $(\alpha=.87, M=5.04, S D=1.10)$ and sexually transmitted IDTs $(\alpha=.88, M=4.88, S D=1.18)$.

\section{External-Attribution-Dependent (EAD) Emotion}

Participants were asked to rate the extent to which they were likely to feel each discrete EAD emotion (if they were in the situation) (e.g., Jin et al., 2016; Jin et al., 2014; van der Meer \& Jin, 2020), on a 7-point Likert scale ranging from " 1 = very unlikely" to " $7=$ very likely." An index of EAD emotion was created by computing anger $(M=4.48, S D=1.95)$, sadness $(M=4.61, S D=1.81)$, surprise $(M=4.14, S D=1.84)$, and confusion $(M=4.40, S D=1.81)$ for respiratory IDT $(\alpha=.83, M=4.41, S D=1.50)$.

An index of EAD emotion for sexually transmitted IDT was also created by averaging anger $(M=4.50, S D=1.91)$, sadness $(M=4.42, S D=1.87)$, surprise $(M=3.81, S D=1.85)$, and confusion $(M=3.98, S D=1.90)(\alpha=.86, M=4.18, S D=1.57)$.

\section{Internal-Attribution-Dependent (IAD) Emotion}

Participants were asked to rate the extent to which they were likely to feel the emotion of "hope" (the IAD emotion identified, see Jin et al., 2016; van der Meer \& Jin, 2020), as measured on a 7-point Likert scale ranging from " $1=$ very unlikely" to " $7=$ very likely" for respiratory $(M=3.44, S D=1.78)$ and sexually transmitted IDTs $(M=3.75, S D=1.81)$. 


\section{Data Analyses}

A General Linear Model (GLM) for Repeated Measures and a path analysis using a model-fit approach through Amos 24 were used to answer the study's research questions and test proposed hypotheses. This study conducted a path analysis because the hope emotion was the only item that did not fit with the requirement of structural equation modeling. Structural equation modeling can use "at least two measured variables as indicators of the latent variable" (Meyers et al., 2013, p. 976).

\section{Results}

Based on the within-subjects experimental design, a GLM for Repeated Measures was run to examine how participants' IDT appraisal (i.e., perceived predictability, controllability, and responsibility) and protective action taking intentions differed as a function of IDT type (i.e., respiratory vs. sexually transmitted). Mediation models through path analysis using a model-fitting approach examined the hypothesized roles of EAD and IAD emotion in mediating the relationship between IDT appraisal and protective action taking intention.

\section{Perceived Threat Appraisal by IDT Type}

RQ1.1 asked whether and how college students' perceived IDT predictability differed by IDT type (i.e., respiratory versus STI). Results showed significant within-subject effects of IDT type on predictability, $F(1,841)=38.36, p \leq .001$, partial $\eta^{2}=.04$. Participants perceived the sexually transmitted IDT as significantly more predictable $(M=4.72, S E=.05)$ than the respiratory $(M=4.45, S E=.05)$.

RQ1.2 asked whether and how college students' perceived IDT controllability might differ by IDT type (i.e., respiratory vs. STI). Significant within-subject effects of IDT type on controllability were detected, $F(1,841)=20.17, p \leq .001$, partial $\eta^{2}=.02$. Participants perceived the sexually transmitted IDT as significantly more controllable $(M=4.82, S E=.05)$ than the respiratory IDT $(M=$ 4.62, $S E=.05)$. 
RQ1.3 asked whether and how college students' perceived IDT responsibility might differ by IDT type (i.e., respiratory versus STI). Significant within-subject effects of IDT type on responsibility were evident, $F(1,841)=54.20, p \leq .001$, partial $\eta^{2}=.06$. Participants perceived higher combined responsibility (i.e., individuals; health professionals; government health organizations at local, state, and federal levels) for the sexually transmitted IDT $(M=3.85, S E=.05)$ than the respiratory IDT $(M=3.50, S E=.06)$.

\section{Differences in Protective Action Taking Intention by IDT Type}

RQ2 asked whether and how college students' intention to take protective actions might differ by IDT type (i.e., respiratory versus STI). Results showed a significant within-subject effects of IDT type on intention to take protective action, $F(1,841)=29.05$, $p \leq .001$, partial $\eta^{2}=.03$. Specifically, individuals had significantly higher intention to take protective actions for the respiratory IDT $(M=5.04, S E=.04)$ than for the sexually transmitted IDT $(M=4.88, S E=.04)$.

\section{Sequential Mediation Models by IDT Type}

According to the concept of emotional flow (Nabi, 2015), people's emotions shift when they are exposed to health messages. Additionally, in terms of attributed-based emotions (Jin et al., 2014), this study further argues people's emotional shifts occur when they attribute the responsibility for IDTs. To understand how emotional flow impacts the relationship between IDT appraisal and behavioral intentions, we examined the proposed sequential mediator role of EAD emotions (i.e., angry, sad, surprised, confused) and an IAD emotion (e.g., hope), respectively, in the relationship between college students' IDT appraisal and protective action taking intentions for respiratory $\left(\mathrm{H}_{1}\right)$ and sexually transmitted IDTs ( $\left.\mathrm{H}_{2}\right)$. To do so, we ran mediation models through path analysis using the model-fit approach, which rendered the following results by IDT type. 


\section{Respiratory IDT}

A variety of goodness-of-fit indices indicated that the estimated model fit the observed data, $\chi^{2}(4, N=842)=37.18, p \leq .001$, with root mean square error of approximation $($ RMSEA $)=.099$, which was higher than the acceptable good fit cutoff of .06 ( Hu \& Bentler, 1999), but less than .10; comparative fit index $(\mathrm{CFI})=.97$; goodness-of-fit index $(\mathrm{GFI})=.99$; and normed-fit index $(\mathrm{NFI})=$ .97. The results indicated a good fit for the mediation model.

There were several significant results rendered by the path analysis of using a model-fit approach. First, the significant results from the standardized regression weights showed that perceived IDT predictability $(\beta=.17, p \leq .001)$ and perceived IDT responsibility $(\beta=.09, p \leq .001)$ were positive predictors for EAD emotions, although perceived IDT controllability $(\beta=.05$, n.s. $)$ was not a significant predictor. Second, EAD emotions (i.e., anger, sadness, surprise, confusion) were a significant positive predictor $(\beta=1.24$, $p \leq .001$ ) for the subsequent IAD emotion (i.e., hope). Lastly, the IAD emotion (i.e., hope) was a significant positive predictor $(\beta=$ $.93, p \leq .001$ ) for participants' protective action taking intentions. In sum, the mediation model showed that for the respiratory IDT type, EAD and IAD emotions, respectively, functioned as sequential mediators for the relationship between two key dimensions: participants' IDT appraisal (perceived predictability and responsibility) and their intention to take protective actions (see Figure 1). Therefore, $\mathrm{H}_{1}$ was supported.

\section{FIGURE 1 Sequential Mediation Model for Respiratory IDT}

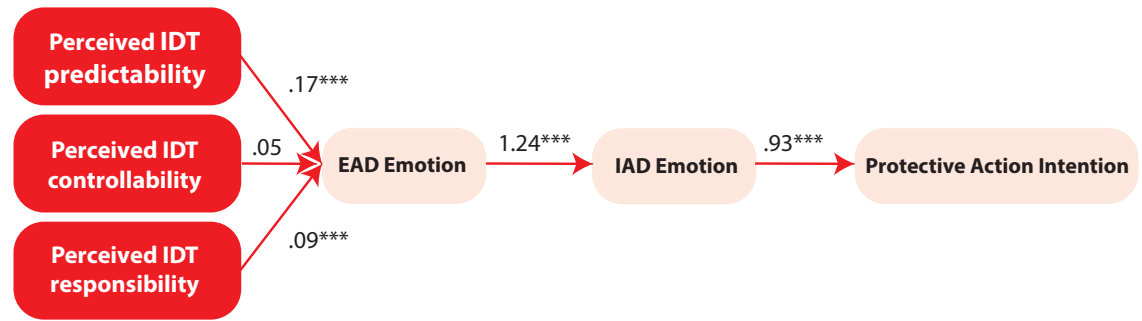

Note. (1) Overall model fit, $\chi^{2}(4, N=842)=37.18, p \leq .001, \mathrm{CFI}=.97$, GFI $=.99$. $\mathrm{NFI}=.97, \mathrm{RMSEA}=.099$. Significant level, ${ }^{\star} p \leq .05,{ }^{* *} p \leq .01,{ }^{* *} p \leq .001$. (2) EAD emotion is an index by computing anger, sadness, surprise, and confusion. IAD emotion is hope. 


\section{Sexually Transmitted IDT}

A variety of goodness-of-fit indices indicated that the estimated model fit the observed data, $\chi^{2}(4, N=842)=27.77, p \leq .001$, with RMSEA $=.08$, which was higher than the acceptable good fit cutoff of .o6 (Hu \& Bentler, 1999), but less than .10; CFI = .98; GFI = .99 ; and NFI $=.98$. Results indicated a good fit for the mediation model.

Several significant results were rendered by the path analysis using a model-fit approach. First, the results from the standardized regression weights showed that perceived IDT predictability $(\beta=.10, p \leq .01)$, controllability $(\beta=.15, p \leq .001)$ and responsibility $(\beta=.09, p \leq .001)$ were significant positive predictors for EAD emotions (i.e., anger, sadness, surprise, confusion). Second, EAD emotions were found to significantly and positively predict $(\beta=.92, p \leq .001)$ the subsequent IAD emotion (i.e., hope). Lastly, the IAD emotion significantly and positively predicted $(\beta=1.30$, $p \leq .001)$ protective action taking intention. In sum, for the sexually transmitted IDT type, the mediation model showed that EAD and IAD emotions, respectively, functioned as sequential mediators for the relationship between all three dimensions of participants' IDT appraisal (i.e., perceived predictability, controllability, and responsibility) and their intention to take protective actions (see Figure 2). Therefore, $\mathrm{H}_{2}$ was supported.

\section{FIGURE 2 Sequential Mediation Model for Sexually Transmitted IDT}

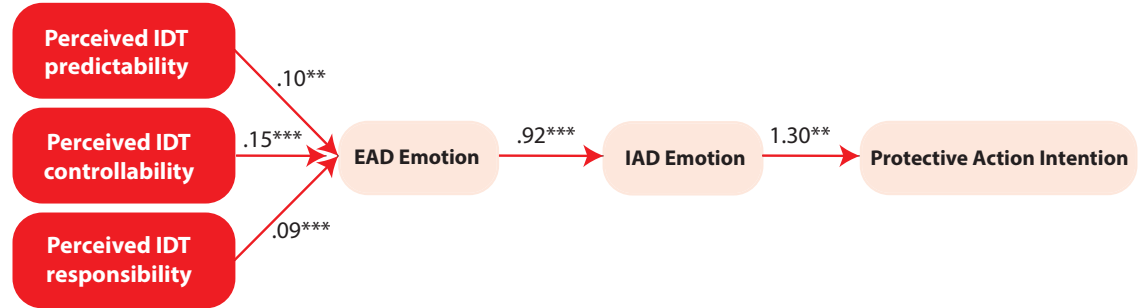

Note. (1) Overall model fit, $\chi^{2}(4, N=842)=27.77, p \leq .001$, CFI $=.98$, GFI $=.99$. $\mathrm{NFI}=.98, \mathrm{RMSEA}=.08$. Significant level, ${ }^{\star} p \leq .05,{ }^{* *} p \leq .01,{ }^{* *} p \leq .001$. (2) EAD emotion is an index by computing anger, sadness, surprise, and confusion. IAD emotion is hope. 


\section{Discussion}

This study was launched and completed at two large research universities in the U.S. prior to the coronavirus (COVID-19) pandemic. Our findings provide a picture of how college students respond cognitively, affectively, and behaviorally to two hypothetical IDT situations, in which a respiratory disease and a STI were spreading and threatening students' health and safety. These findings are valuable baseline evidence that add to the relatively scarce literature on IDT communication on college campuses despite its critical importance. Besides its practical value to health communicators, our study also contributes to risk and crisis communication theory by advancing the new IDT appraisal model (Jin et al., 2020) with a college student sample's responses to two distinct IDTs.

\section{Challenges and Opportunities for Sexually Transmitted IDT Communication}

Our college student participants perceived STIs as significantly more predictable and more controllable than respiratory IDTs. These findings imply that college students tend to feel familiar with and sufficiently educated about STIs, probably due to public health information available on campus and via various channels as well as the availability of vaccines (e.g., HPV vaccine). The transmission mode of STIs is also likely to be perceived as more personally controllable than respiratory infections. This relatively higher sense of personal control over STI risk exposure may explain the higher level of blame our participants assigned to responsible parties for STI situations. It seems that for college students, STI causes and transmission routes are broadly familiar. Therefore, college students expect STI threats should be taken care of by people who themselves are at risk, together with health professionals and health agencies to control and prevent disease transmission.

However, compared to their responses to the respiratory IDT, our college student participants reported significantly lower levels of intentions to take protective actions against the STI. This finding echoes prior research on optimistic bias among college students (e.g., Afifi \& Weiner, 2006; Best et al., 2018), which occurs when 
individuals disconnect their disease prevention knowledge from preventative behaviors they can take. Therefore, when it comes to communicating about STIs to college students, the emphasis should lie in (a) conquering the optimistic bias caused unintentionally by existing knowledge and false confidence in one's invincibility, (b) motivating college students to take preventive actions as early as possible, and (c) shifting the blame from others for disease prevention and control responsibility to having students take personal responsibility for a controllable event.

\section{Challenges and Opportunities for Respiratory IDT Communication}

Before the COVID-19 pandemic, our college student participants perceived a respiratory IDT as significantly less predictable and less controllable than a STI. This indicates that the transmission mode of the disease itself evokes high uncertainty and higher risk perception, compared to STIs. We speculate that the unpredictability and uncontrollability of respiratory IDTs are perceived as even higher now given the COVID-19 situation and the high uncertainty as well as high inconsistency of COVID-19 communication (e.g., Bogel-Burroughs, 2020; Kafka, 2020).

Our participants also perceived lower levels of IDT responsibility (or much less blame assigned to at-risk individuals, health professionals, and government health organizations) for the respiratory IDT than for the sexually transmitted IDT. This finding sheds light on the importance of providing timely and accurate information on the disease itself and who is in charge of disease prevention and control (Seeger, 2006), as well as how college students themselves can participate effectively and confidently in the prevention process (Lee et al., 2018). For example, to increase adherence to protective behaviors against the spread of COVID19, experts have encouraged decision makers to "use clear, consistent, and transparent messaging" and to "foster a sense of efficacy and avoid fatalism" (National Research Council, 2020, p. 1). In light of current COVID-19 crisis and future respiratory IDTs, university leaders and health officers should focus on lowering uncertainty (thus increasing college students' perceived predictability 
and controllability of the situation). For example, they can provide information about free or low cost COVID-19 testing, contact tracing, and other efforts to mitigate the disease threat.

University leaders and health officers also can foster a sense of self-responsibility among college students so that they can better protect themselves and help protect others when possible. For example, communication may convey information regarding social disapproval of failure to comply with recommended guidelines for disease prevention, such as wearing masks and maintaining social distancing, and strategies to present preventative behaviors as part of students' habitual responsibilities (e.g., placing hand sanitizer near the door and encouraging hand sanitizing each time people enter the room) (National Research Council, 2020).

Compared to sexually transmitted IDT responses, our participants indicated significantly higher intentions to take protective actions against a respiratory IDT. For health communicators and university leaders, this high motivation for self-protection is a solid base for effective respiratory IDT (e.g., COVID-19) communication. To capitalize on the momentum of college students' desire to take timely and recommended actions, IDT messages should provide accurate information from credible sources, clear instructions conveyed in concise and engaging language, and provide consistent recommendations for college students to follow, as evidenced in prior research on HPV (Nabi \& Prestin, 2016) and flu-vaccine communication (Lee et al., 2018) among college students. It might also be helpful to encourage college students to share information such as how to take proper protective actions with their peers who likely have similar respiratory IDT appraisals and need similar cognitive and behavioral support.

\section{Strategic Value of Emotional Sequencing in IDT Communication}

Across the two types of IDTs, we observed similar patterns that suggest the strategic value of emotional sequencing for IDT strategic communication. First, as Jin et al. (2014) argued, emotions and measures of emotions are highly context-specific. Built upon existing emotions identified by crisis and health scholars (e.g., Jin, 
et al., 2016; van der Meer \& Jin, 2020), our study advocates for the importance of identifying emotions and affective responses that are most relevant and especially pertinent to IDTs. As the first attempt, we focused on attribution-dependent emotions and created EAD and IAD IDT emotion clusters. For both IDTs, the "IDT appraisal $\rightarrow$ EAD emotion $\rightarrow$ IAD emotion $\rightarrow$ behavioral intention" sequential mediation model was supported. Such findings imply that, when confronted by these two IDT types, college students' IDT appraisal will first lead to externalattribution-triggered affect, which can be a combination of anger, sadness, surprise, and confusion. From there, such negative emotions triggered by the situation motivate individuals to take control of the situation and foster a sense of hope, which subsequently triggers increased intentions to take protective actions. This observed pattern highlights the power of emotions in health risk and crisis communication and how affect, even when negative, can be channeled into a positive force (e.g., hope) that is futureoriented. Such future-oriented mental and emotional positions seem to pave a promising path for understanding college students' protective action taking.

Comparing the two mediation models, we observed some differences in the way college students appraise respiratory and sexually transmitted IDTs. For the sexually transmitted IDT, all three appraisal dimensions (i.e., predictability, controllability, responsibility) tended to be positive predictors of EAD emotions. However, for the respiratory IDT, only predictability and responsibility led to EAD emotions. Perceived controllability of a respiratory IDT does not seem to impact EAD emotions, which merits future research to provide further evidence-based explanations.

The insights from our sequential mediation models, centering on the critical "connector" role different IDT emotions (EAD and IAD) play in triggering desired behavioral outcomes, provide practical recommendations for health communicators. To be effective and relatable to college students, IDT communication messages need to convey relevant information that is emotionally engaging and motivates individuals to make the connection between IDT appraisals and protective action taking. In addition to having only one focal emotion as the affective appeal throughout an 
IDT communication campaign, health communicators can consider identifying multiple primary emotions to be embedded in their messages to first alert college students and then cultivate a sense of optimism and hope to motivate them to take protective actions. This is indeed a mixed-emotions approach for health persuasion, which can address some of the side effects of health risk message over-exposure (e.g., Kinnick et al., 1996; So et al., 2017). For example, in studying AIDS/HIV message effects, Kinnick et al. (1996) found that long-term exposure to issue-related messages led to apathetic feelings and emotional burnout regarding the health issue itself. So et al. (2017) reported that repeated exposure to obesity-related messages made individuals feel exhausted and bored, which consequently made them disengaged from and resistant to be involved in future messages regarding the same issue. By sequencing emotional appeals and anticipating sequenced affective responses, an IDT prevention message can avoid (a) creating emotional fatigue and (b) affective overload.

\section{Limitations and Future Directions}

This study has several limitations. First, survey participants were college students from two U.S. universities. Thus, the findings are not generalizable to other college students in other universities or in other countries or cultural contexts.

Second, we only examined two IDT types differentiated by the mode of transmission. How college students respond to other IDTs (e.g., foodborne, waterborne, and vector-borne) needs to be examined in the future.

Third, to improve the new IDT appraisal model, the interconnection and mutual influence between the three IDT dimensions (i.e., predictability, controllability, and responsibility) should be further investigated.

Fourth, this study only measured emotional responses to attribution-dependent crisis emotions. Future studies should measure attribution-independent crisis emotion as well as investigate how crisis emotions form and evolve over time. Future research also should investigate how to maximize emotions' role in motivating behavioral changes. 
Fifth, this study includes self-reported data, but the study design could only measure individuals' intentions to take preventive action instead of actual behavior. Thus, future studies should consider conducting longitudinal research, as funding allows, to examine how people take action in response to IDTs over time.

Sixth, the study did not measure participants' motivations to think about IDTs. The survey was not conducted during a flu season or amid the COVID-19 pandemic, and thus the stimulus outbreak messages may not have triggered as much motivations to read and respond to as health messages seen during serious IDT situations. Building on the model tested in this study, future research should consider devising more naturalistic study designs and adding motivational variables into the IDT appraisal model.

Seventh, our study did not ask participants whether they were sexually active. Future research should include such a measure and examine how the varied levels of college students' sexual activeness might impact their risk and threat perceptions. The history of STIs was not captured in our study but could have influenced our findings in terms of an optimism bias.

Lastly, how the COVID-19 pandemic and universities' various communication efforts have impacted current and future college students' responses to respiratory and other IDT types overall merits ongoing assessment and longitudinal examination. In particular, the COVID-19 pandemic is a unique opportunity to examine how college students and others respond to ever-present IDTs (e.g., STIs) during rarely-occurring threats (e.g., respiratory diseases).

\section{Conclusion}

Universities face frequent crises including natural disasters, active shooter incidents, scandals, and infectious disease outbreaks (Moerschell \& Novak, 2020). A broad body of scholarship informs how college students cope with STIs (e.g., Lin \& Lagoe, 2013; Vorpahl \& Yang, 2018; Yang, 2015), but less is known about respiratory IDTs. Findings from this study contribute to our limited understanding of how college students respond to respiratory 
IDTs, compared to STIs. In turn, these findings inform public health messaging during outbreaks.

Importantly, our findings indicate that communication about STIs must combat optimistic bias through early and repeated interventions that focus on students' personal responsibility for a controllable, but frequent risk. Communication about respiratory IDTs should focus on lowering uncertainty, thereby increasing students' efficacy to reasonably combat less frequent threats.

Communication about respiratory IDTs also must harness college students' elevated intentions to take protective actions through providing clear, credible, consistent, and engaging information about the best mitigation actions to protect themselves and others. Communication about both IDT types needs to be cognitively relevant to students, emotionally engaging in a positive way, and motivating to connect students' threat appraisals to reasonable protective actions they can take. While crises are frequent on college campuses, effective risk and crisis communication can mitigate negative outcomes.

\section{ORCID}

Yan Jin (i) https://orcid.org/oooo-0oo3-4228-5529

Yen-I Lee (ㄱ) https://orcid.org/oooo-00o1-7820-6568

Brooke Fisher Liu (1) https://orcid.org/0ooo-0003-1985-8050

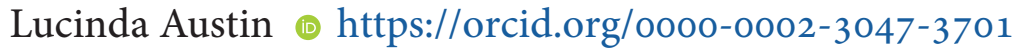

Seoyeon Kim 사 https://orcid.org/oooo-0002-2018-9520

\section{References}

Afifi, W. A., \& Weiner, J. L. (2006). Seeking information about sexual health: Applying the theory of motivated information management. Human Communication Research, 32(1), 25-57. https://doi.org/10.1111/j.1468-2958.2006.00002.x

Agarwal, V. (2014). A/ $\mathrm{H}_{1} \mathrm{~N}_{1}$ vaccine intentions in college students: An application of the theory of planned behavior. Journal of American College Health, 62(6), 416-424. https://doi.org/10.1 080/07448481.2014.917650 
Alsulaiman, S. A., \& Rentner, T. L. (2018). The health belief model and preventive measures: A study of the Ministry of Health campaign on coronavirus in Saudi Arabia. Journal of International Crisis and Risk Communication Research, 1(1), 27-56. https://doi.org/10.30658/jicrcr.1.1.3

Barnett, T., Fournié, G., Gupta, S., \& Seeley, J. (2015). Some considerations concerning the challenge of incorporating social variables into epidemiological models of infectious disease transmissions. Global Public Health, 10(4), 438-448. https:// doi.org/10.1080/17441692.2015.1007155

Best, A. L., Logan, R. G., Vazquez-Otero, C., Fung, W., Chee, V., Thompson, E. L., Villalona, S., Thompson, L. M. A., Gwede, C. K., \& Daley, E. M. (2018). Application of a health literacy framework to explore patients' knowledge of the link between HPV and cancer. Journal of Health Communication, 23(8), 695-702. https://doi.org/10.1080/10810730.2018.1508257

Bogel-Burroughs, N. (2020, January 31). Masks are on. Games are canceled. Fear of the coronavirus comesto U.S.colleges. New York Times. https://web.archive.org/web/20200131101233/https:// www.nytimes.com/2020/01/31/us/coronavirus-collegecampuses.html

Boudewyns, V., \& Paquin, R. S. (2011). Intentions and beliefs about getting tested for STDs: Implications for communication interventions. Health Communication, 26(8), 701-711. https://doi.org/10.1080/10410236.2011.563353

Brummette, J., \& Sisco, H. F. (2015). Using Twitter as a means of coping with emotions and uncontrollable crises. Public Relations Review, 41(1), 89-96. https://doi.org/10.1016/j. pubrev.2014.10.009

Burke, L. (2020). Communicating through a crisis. Inside Higher Education. https://web.archive.org/web/20201106083305/ https://www.insidehighered.com/news/2020/11/06/communications-research-suggests-leaders-think-aboutcovid-19-differently-other

Centers for Disease Control and Prevention (CDC). (n.d.). Tips for College Health and Safety. https://www.cdc.gov/health equity/features/college/ 
Cheah, W. H. (2006). College students' perspectives in sexually transmitted infections (STIs) in Malaysia, Singapore, England, and the U.S.: Formative evaluation insights and implications. Journal of Intercultural Communication Research, 35(3), 219233. https://doi.org/10.1080/17475750601026974

Choi, Y., \& Lin, Y. H. (2009). Consumer responses to Mattel product recalls posted on online bulletin boards: Exploring two types of emotion. Journal of Public Relations Research, 21(2), 198-207. https://doi.org/10.1080/10627260802557506

Coombs, W. T. (1998). An analytic framework for crisis situations: Better responses for a better understanding of the situation. Journal of Public Relations Research, 10(3), 177-191. https:// doi.org/10.1207/s1532754xjprr1003_02

Coombs, W. T., \& Holladay, S. J. (2005). An exploratory study of stakeholder emotions: Affect and crises. In N. M. Ashkanasy, W. J. Zerbe, \& C. E. J. Härtel (Eds.), The Effect of Affect in Organizational Settings: Research on Emotion in Organizations (Vol. 1, pp. 263-280). Emerald Group Publishing Limited. https://doi.org/10.1016/S1746-9791(05)01111-9

Cornally, N., Deasy, E. A., McCarthy, J. M., \& Weathers, E. (2013). Student nurses' intention to get the influenza vaccine. British Journal of Nursing, 22(21), 1207-1211. https://doi. org/10.12968/bjon.2013.22.21.1207

Cowling, B. J., Ng, D. M., Ip, D. K., Liao, Q., Lam, W. W., Wu, J. T., Lau, J. T., Griffiths, S. M., \& Fielding, R. (2010). Community psychological and behavioral responses through the first wave of the 2009 influenza A $\left(\mathrm{H}_{1} \mathrm{~N}_{1}\right)$ pandemic in Hong Kong. The Journal of Infectious Diseases, 202(6), 867-876. https://doi. org/10.1086/655811

de Zwart, O., Veldhuijzen, I. K., Elam, G., Aro, A. R., Abraham, T., Bishop, G. D., Voeten, H. A. C. M., Richardus, J. H., \& Brug, J. (2009). Perceived threat, risk perception, and efficacy beliefs related to SARS and other (Emerging) infectious diseases: Results of an international survey. International Journal of Behavioral Medicine, 16(1), 30-40. https://doi.org/10.1007/ s12529-008-9008-2 
Floyd, D. L., Prentice-Dunn, S., \& Rogers, R. W. (2000). A meta-analysis of research on protection motivation theory. Journal of Applied Social Psychology, 3o(2), 407-429. https:// doi.org/10.1111/j.1559-1816.2000.tbo2323.X

Guo, S. Z., Cheong, A. W. H., \& Shen, C. F. (2005). Depth of reasoning and information processing: A predictive model of SARS behavior. Asian Journal of Communication, 15(3), 274288. https://doi.org/10.1080/01292980500261589

Hu, L. T., \& Bentler, P. M. (1999). Cutoff criteria for fit indexes in covariance structure analysis: Conventional criteria versus new alternatives. Structural Equation Modeling, 6(1), 1-55. https://doi.org/10.1080/10705519909540118

Inungu, J., Mumford, V., Younis, M., \& Langford, S. (2009). HIV knowledge, attitudes and practices among college students in the United States. Journal of Health and Human Services Administration, 32(3), 259-277.

Jin, Y. (2010). Making sense sensibly in crisis communication: How public's crisis appraisals influence their negative emotions, coping strategy preferences, and crisis response acceptance. Communication Research, 37(4), 522-552. https://doi. org/10.1177/0093650210368256

Jin, Y., Fraustino, J. D., \& Liu, B. F. (2016). The scared, the outraged, and the anxious: How crisis emotions, involvement, and demographics predict publics' conative coping. International Journal of Strategic Communication, 4(4), 289-308. https://doi. org/10.1080/1553118X.2016.1160401

Jin, Y., Iles I., Austin, L., Liu, B. F., \& Hancock, G. (2020). The infectious disease threat (IDT) appraisal model: How perceptions of IDT predictability and controllability predict individuals' responses to risks. International Journal of Strategic Communication, 14(4), 246-271. https://doi.org/10.1080/155 3118X.2020.1801691

Jin, Y., Liu, B. F., Anagondahalli, D., \& Austin, L. (2014). Scale development for measuring publics' emotions in organizational crises. Public Relations Review, 4o(3), 509-518. https:// doi.org/10.1016/j.pubrev.2014.04.007 
Jin, Y., Pang, A., \& Cameron, G. T. (2010). The role of emotions in crisis responses: Inaugural test of the integrated crisis mapping (ICM) model. Corporate Communications: An International Journal, 15(4), 428-452. https://doi. org/10.1108/13563281011085529

Kafka, A. C. (2020, March 12). Shock, fear, and fatalism: As coronavirus prompts colleges to close, students grapple with uncertainty. Chronicle of Higher Education. https://web.archive.org/ web/20200812213108/https://www.chronicle.com/article/ shock-fear-and-fatalism-as-coronavirus-prompts-colleges-toclose-students-grapple-with-uncertainty/

Kim, S. C., \& Hawkins, K. H. (2020). The psychology of social media communication in influencing prevention intentions during the 2019 US measles outbreak. Computers in Human Behavior, 111, 106428. https://doi.org/10.1016/j.chb.2020.106428

Kinnick, K. N., Krugman, D. M., \& Cameron, G. T. (1996). Compassion fatigue: Communication and burnout toward social problems. Journalism \& Mass Communication Quarterly, 73(3), 687-707. https://doi.org/10.1177/107769909607300314 Kotalik, J. (2005). Preparing for an influenza pandemic: Ethical issues. Bioethics, 19(4), 422-431. https://doi.org/10.1111/ j.1467-8519.2005.00453.X

Kuang, K., \& Wilson, S. R. (2017). A meta-analysis of uncertainty and information management in illness contexts. Journal of Communication, 67(3), 378-401. https://doi.org/10.1111/ jcom.12299

Lau, J. T. F., Yang, X., Tsui, H., \& Kim, J. H. (2003). Monitoring community responses to the SARS epidemic in Hong Kong: From day 10 to day 62. Journal of Epidemiology \& Community Health, 57(11), 864-870. https://doi.org/10.1136/jech.57.11.864

Lee, S. I. (2015). Costly lessons from the 2015 Middle East respiratory syndrome coronavirus outbreak in Korea. Journal of Preventive Medicine and Public Health, 48(6), 274-276.

Lee, Y-I., \& Jin, Y. (2019). Crisis information seeking and sharing (CISS): Scale development for measuring publics' communicative behavior in social-mediated public health crises. Journal of International Crisis and Risk Communication Research, 2(1), 13-38. https://doi.org/10.30658/jicrcr.2.1.2 
Lee, Y-I., Jin, Y., \& Nowak, G. (2018). Motivating influenza vaccination among young adults: The effects of public service advertising message framing and text versus image support. Social Marketing Quarterly, 24(2), 89-103. https:// doi.org/10.1177/1524500418771283

Leung, G. M., Ho, L-M., Chan, S. K. K., Ho, S-Y., Bacon-Shone, J., Choy, R. Y. L., Hedley, A. J., Lam, T-H., \& Fielding, R. (2005). Longitudinal assessment of community psychobehavioral responses during the 2003 outbreak of severe acute respiratory syndrome in Hong Kong. Clinical Infectious Diseases, 50(12), 1713-1720. https://doi.org/10.1086/429923

Lin, C. A., \& Lagoe, C. (2013). Effects of news media and interpersonal interactions on $\mathrm{H}_{1} \mathrm{~N}_{1}$ risk perception and vaccine intent. Communication Research Reports, 30(2), 127-136. https://doi. org/10.1080/08824096.2012.762907

Liu, B. F., Austin, L., Lee, Y-I., Jin, Y., \& Kim, S. (2020). Telling the tale: The role of narratives in helping people respond to crises. Journal of Applied Communication Research, 48(3), 328-349. https://doi.org/10.1080/00909882.2020.1756377

Liu, B. F., Fraustino, J., \& Jin, Y. (2016). Social media use during disasters: How information form and source influence intended behavioral responses. Communication Research, 43(5), 626-646. https://doi.org/10.1177/0093650214565917

Liu, W., Lai, C-H., \& Xu, W. (2018). Tweeting about emergency: A semantic network analysis of government organizations' social media messaging during Hurricane Harvey. Public Relations Review, 44(5), 807-819. https://doi.org/10.1016/j. pubrev.2018.10.009

Meyers, L. S., Gamst, G., \& Guarino, A. J. (2013). Applied multivariate research ( 2 nd ed.). Sage.

Moerschell, L., \& Novak, S. S. (2020). Managing crisis in a university setting: The challenge of alignment. Journal of Contingencies and Crisis Management, 28(1), 30-40. https:// doi.org/10.1111/1468-5973.12266

Nabi, R. L. (2015). Emotional flow in persuasive health messages. Health Communication, 30, 114-124. https://doi.org/10.1080/ 10410236.2014.974129 
Nabi, R. L., \& Myrick, J. G. (2019). Uplifting fear appeals: Considering the role of hope in fear-based persuasive messages. Health Communication, 34(4), 463-474. https://doi.org/ 10.1080/10410236.2017.1422847

Nabi, R. L., \& Prestin, A. (2016). Unrealistic hope and unnecessary fear: Exploring how sensationalistic news stories influence health motivation. Health Communication, 31(9), 1115-1126. https://doi.org/10.1080/10410236.2015.1045237

National Research Council. (2020). Encouraging adoption of protective behaviors to mitigate the spread of COVID-19: Strategies for behavior change. The National Academies Press. https:// doi.org/10.17226/25881

Panda, P., Chakraborty, A., \& Dror, D. M. (2015). Mobilizing community-based health insurance to enhance awareness and prevention of airborne, vectorborne, and waterborne diseases in rural India. Indian Journal of Medical Research, 142(2), 151164. https://doi.org/10.4103/0971-5916.164235

Pickles, H., \& Goodwin, R. (2006). Pandemic flu: Encouraging a positive population response. Eurohealth, 12(1), 10-12.

Rintamaki, L. S., \& Yang, Z. J. (2013). Advancing the extended parallel process model through the inclusion of response cost measures. Journal of Health Communication, 19(7), 759-774. https://doi.org/10.1080/10810730.2013.864722

Roberto, A. J., Mongeau, P. A., Liu, Y., Hashi, E. C. (2019). “Fear the flu, not the flu shot": A test of the extended parallel process model. Journal of Health Communication, 24(11), 829-836. https://doi.org/10.1080/10810730.2019.1673520

Ropeik, D. (2002). Understanding factors of risk perception. Nieman Reports, 56(4), 52. https://niemanreports.org/articles/ understanding-factors-of-risk-perception/

Rubin, G. J., Amlǒt, R., Page, L., \& Wessely, S. (2009). Public perceptions, anxiety, and behavior change in relation to the swine flu outbreak: Cross sectional telephone survey. BMJ, 339(b2651), 1-8. https://doi.org/10.1136/bmj.b2651

Schram, J. (2003). How popular perceptions of risk from SARS are fermenting discrimination. British Medical Journal, 326(7395), 939. https://doi.org/10.1136/bmj.326.7395.939 
Seeger, M. W. (2006). Best practices in crisis communication: An expert panel process. Journal of Applied Communication Research, 34(3), 232-244. https://doi.org/10.1080/00909880 600769944

Slovic, P. (1987). Perception of risk. Science, 236(4799), 280-285. https://doi.org/10.1126/science.3563507

Smith, R. D. (2006). Responding to global infectious disease outbreaks: Lessons from SARS on the role of risk perception, communication and management. Social Science \& Medicine, 63(12), 3113-3123.

Snyder, C. R., Sympson, S. C., Ybasco, S. F. C., Borders, T. F., Babyak, M. A., \& Higgins, R. L. (1996). Development and validation of the State Hope Scale. Journal of Personality and Social Psychology, 7o(2), 321-335. https://doi.apa.org/ doi/10.1037/0022-3514.70.2.321

So, J., Kim, S., \& Cohen, H. (2017). Message fatigue: Conceptual definition, operationalization, and correlates. Communication Monographs, 84(1), 5-29. https://doi.org/10.1080/03637751. 2016.1250429

Taha, S. A., Matheson, K., \& Anisman, H. (2013). H1N1 was not all that scary: Uncertainty and stressor appraisals predict anxiety related to a coming viral threat. Stress Health, 30(2), 149-157. https://doi.org/10.1002/smi.2505

van der Meer, T. G. L. A., \& Jin, Y. (2020). Seeking formula for misinformation treatment in public health crises: The effects of corrective information type and source. Health Communication, 35(5), 560-575. https://doi.org/10.1080/10410236.2019.1573 295

Vorpahl, M. M., \& Yang, J. Z. (2018). Who is to blame? Framing $\mathrm{HPV}$ to influence vaccination intentions among college students. Health Communication, 33 (5), 620-627. https://doi.org/ 10.1080/10410236.2017.1289436

Washer, P. (2004). Representations of SARS in the British newspapers. Social Science \& Medicine, 59(12), 2561-2571. https:// doi.org/10.1016/j.socscimed.2004.03.038 
Weiner, B. (1986). Attribution, emotion, and action. In R. M. Sorrentino \& E. T. Higgins (Eds.), Handbook of Motivation and Cognition: Foundations of Social Behavior (pp. 281-313). Guilford.

Weinstein, N. D. (1984). Why it won't happen to me: Perceptions of risk factors and susceptibility. Health Psychology, 3(5), 431457. https://doi.org/10.1037//0278-6133.3.5.431

Witte, K. (1992). Putting the fear back into fear appeals: The extended parallel process model. Communication Monographs, 59(4), 329-349. https://doi.org/10.1080/03637759209376276

Yang, Z. J. (2015). Predicting young adults' intentions to get the $\mathrm{H}_{1} \mathrm{~N}_{1}$ vaccine: An integrated model. Journal of Health Communication, 2o(1), 69-79. https://doi.org/10.1080/10810 730.2014.904023

Yang, Z. J., \& Pittman, M. M. (2017). The silver lining of shame: Framing HPV vaccination intentions. Health Communication, 32(8), 987-994. https://doi.org/10.1080/10410236.2016.11964 20

Zhang, L., Kong, Y., \& Chang, H. (2015). Media use and health behavior in $\mathrm{H}_{1} \mathrm{~N}_{1}$ flu crisis: The mediating role of perceived knowledge and fear. Atlantic Journal of Communication, 23(2), 67-8o. https://doi.org/10.1080/15456870.2015.1013101

Zhang, Y., Jin, Y., \& Tang, Y. (2015). Framing depression: Cultural and organizational influences on coverage of a public health threat and attribution of responsibilities in Chinese news media, 2000-2012. Journalism \& Mass Communication Quarterly, 92(1), 99-120. https://doi.org/10.1177\%2F1077699 014558553 